\title{
Paper
}

\section{Numerical Investigation on Crack Detection Technique for Electric Connector with Joule Heating Effect}

\author{
Thomas Jin-Chee LiU*† Member
}

(Received May 4, 2021, revised October 8, 2021)

\begin{abstract}
Using the electro-thermo-structural coupled-field finite element analysis, the crack detection technique with the Joule heating effect for the electric connector is studied and discussed. The physical behavior of the electric connector with an edge crack is investigated numerically. The electric current density, Joule heating temperature and structural deformation are obtained. As a result, the electric current concentration and Joule heating effect can induce a local hot spot or hot band region around the crack tip. Furthermore, the crack tip location can be determined according to the temperature distribution. For the accurate crack detection, two better electric loading conditions are found as follows: (a) shorter electric operating time $\left(t_{p}\right)$, (b) larger electric current $\left(I_{0}\right)$. The optimal condition, $I_{0}=100 \mathrm{~A}$ and $t_{p}=0.001 \mathrm{~s}$, is found for this crack detection research.
\end{abstract}

Keywords: Electric connector, Electro-thermo-structural, Joule heating, Crack detection, Finite element,

\section{Introduction}

The electric connector as shown in Fig. 1 is widely used in electronic products such as mobile phones, cameras and laptop computers. When the connector touches the electrode, the electric current or signal can be transferred through the contact surface. The electric connector is usually designed as an elastic spring to keep the electric contact on the connector/electrode interface. Under the working condition, the connector is subjected to the electro-thermostructural coupling loads.

The strength and reliability of the electric connector are very important so that the existence of the crack or defect must be avoided or controlled. The cracks may exist in the electric connector after long working period. If a crack is found, the unstable crack growth must be avoided. The cracked electric connector must be replaced by a new one to keep a good working condition.

The crack detection technique is an important issue for the structural health and strength of the electric connector. The Joule heating method [1] [3] is one of the crack detection technique and can be applied on the electric connector. Many researches have reported that the Joule heating effect can induce the local hot spot at the crack tip [1] [5]. As shown in Fig. 2, when the crack tip is subjected to the electric load, the electric current density field concentrates at the crack tip. Due to the Joule heating, the electric concentration causes the hot spot at the crack tip zone. The crack tip location can be determined according to the hot spot, and the crack detection is achieved.

In this paper, the physical behavior of the electric con-

\footnotetext{
* Corresponding: jinchee@mail.mcut.edu.tw

$\dagger$ Department of Mechanical Engineering

Ming Chi University of Technology, Taishan, New Taipei City, Taiwan
}

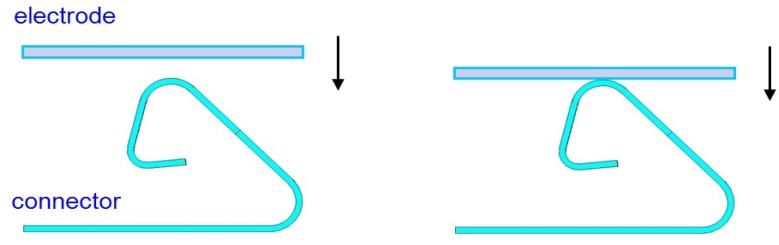

Figure 1: Typical electric connector.
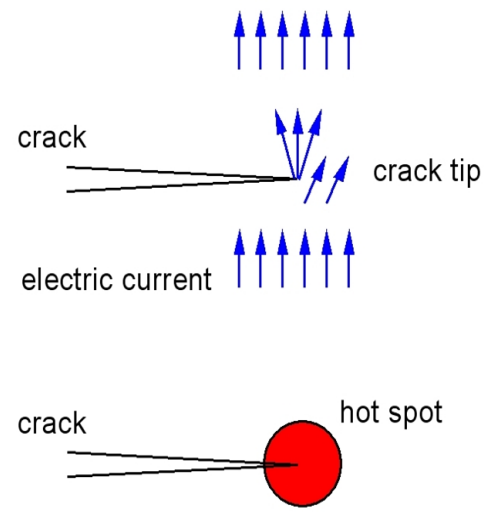

Figure 2: Hot spot at crack tip.

nector with an edge crack is investigated using the electrothermo-structural coupled-field finite element analysis. The electric current density, Joule heating temperature and structural deformation are obtained. Especially, the temperature field and hot region around the crack tip are discussed. Furthermore, the crack detection technique with the Joule heating effect is numerically investigated. 


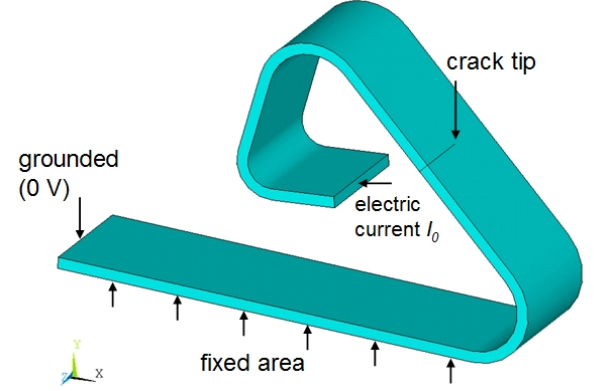

Figure 3: Case study of this research.
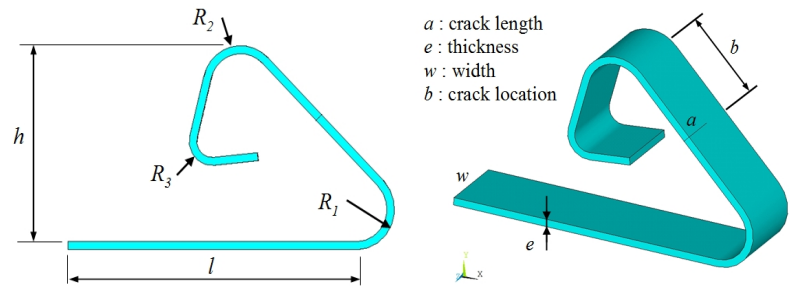

Figure 4: Geometry of this research.

Table 1: Material data of UNS C17000 beryllium copper [6].

\begin{tabular}{ll}
\hline density & $8260 \mathrm{~kg} / \mathrm{m}^{3}$ \\
thermal conductivity & $118 \mathrm{~W} / \mathrm{m}-\mathrm{K} @ 20^{\circ} \mathrm{C}$ \\
& $145 \mathrm{~W} / \mathrm{m}-\mathrm{K} @ 200^{\circ} \mathrm{C}$ \\
specific heat & $420 \mathrm{~J} / \mathrm{kg}-\mathrm{K}$ \\
resistivity & $1^{*} 10^{-7} \Omega-\mathrm{m}$ \\
coefficient of thermal & $1.67 * 10^{-5} 1{ }^{\circ} \mathrm{C} @ 20 \sim 100{ }^{\circ} \mathrm{C}$ \\
expansion & $1.70^{*} 10^{-5} 1 /{ }^{\circ} \mathrm{C} @ 200{ }^{\circ} \mathrm{C}$ \\
& $1.78^{*} 10^{-5} 1 /{ }^{\circ} \mathrm{C} @ 300^{\circ} \mathrm{C}$ \\
Young's modulus & $115 \mathrm{GPa}$ \\
Poisson's ratio & 0.3 \\
Yielding strength & $190 \mathrm{MPa}$ \\
tangent modulus & $11.5 \mathrm{GPa}$ \\
melting point & $865-980{ }^{\circ} \mathrm{C}$ \\
\hline
\end{tabular}

\section{Problem Definition and Finite Element Analysis}

In Fig. 3 and Fig. 4, it shows the sample geometry, boundary condition and electric load of this research. The crack length is denoted as $a$. The sample is subjected to a direct current (DC) $I_{0}$. The material type of the sample is the UNS C17000 beryllium copper. In Table 1 , it lists the material data [6].

The following conditions are considered in this study:

(1) Three-dimensional analysis

(2) Electro-thermo-structural coupled-field

(3) Transient heat transfer

(4) Convection on air/sample interface

(5) Steady electric current (DC current)

(6) Temperature-dependent material data

(7) With electric/thermal/mechanical contact condition on crack surface

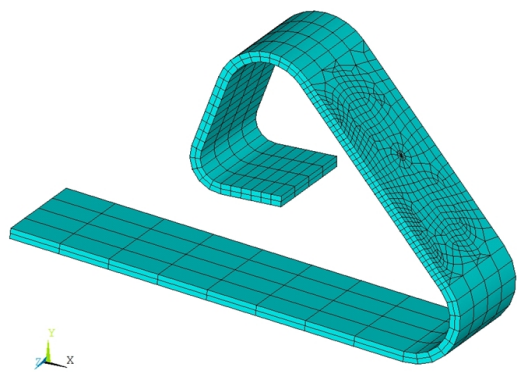

(a) Full model

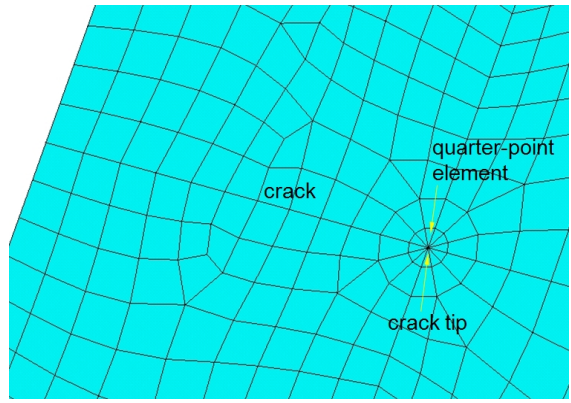

(b) Local mesh around crack

Figure 5: Finite element model.

In this research, the finite element equation of the electrothermo-structural coupled-field analysis is listed as the following matrix-form [7]:

$$
\begin{gathered}
{\left[\begin{array}{ccc}
M & 0 & 0 \\
0 & 0 & 0 \\
0 & 0 & 0
\end{array}\right]\left[\begin{array}{c}
\ddot{U} \\
\ddot{T} \\
\ddot{V}
\end{array}\right]+\left[\begin{array}{ccc}
C & 0 & 0 \\
C^{t u} & C^{t} & 0 \\
0 & 0 & 0
\end{array}\right]\left[\begin{array}{c}
\dot{U} \\
\dot{T} \\
\dot{V}
\end{array}\right]} \\
+\left[\begin{array}{ccc}
K & K^{u t} & 0 \\
0 & K^{t} & 0 \\
0 & 0 & K^{v}
\end{array}\right]\left[\begin{array}{c}
U \\
T \\
V
\end{array}\right]=\left[\begin{array}{c}
F \\
Q \\
I
\end{array}\right]
\end{gathered}
$$

where U, T, V, F, Q and I are the vector forms of the displacement, temperature, electric potential, force, heat flow rate and electric current, respectively. The material constant matrices $\mathrm{M}, \mathrm{C}, C^{t}, C^{t u}, \mathrm{~K}, K^{t}, K^{u t}$ and $K^{v}$ are the structural mass, structural damping, thermal specific heat, thermostructural damping, structural stiffness, thermal conductivity, thermo-structural stiffness and electric conductivity, respectively. The coupled heat flow matrix Q contains the effects of the thermal loading and electrical Joule heating. $C^{t u}$ and $K^{u t}$ are thermo-structural coupled terms. Eq. 1 is a directly coupled nonlinear equation which is solved using the Newton-Raphson iterative method.

Figure 5 shows the finite element model of ANSYS. It is modeled by the SOLID226 element, i.e. the 20-node isoparametric solid element associated with the electrothermo-structural analysis. The crack tip region is modeled by the quarter-point elements for expressing the $r^{-1 / 2}$ singularity of the electric current density field.

The electric/thermal/mechanical contact condition on the crack surfaces is considered in the simulation. The approximate method proposed in [8] [9] is adopted in this study. The ANSYS contact element types: TARGE170 and CONTA174 are utilized to express the electric/thermal/ me- 


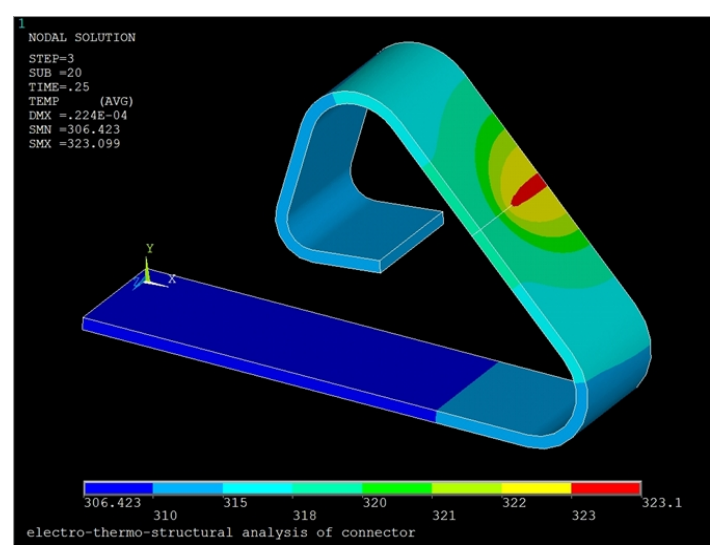

Figure 6: Temperature distribution (units: ${ }^{\circ} \mathrm{C}$ ).

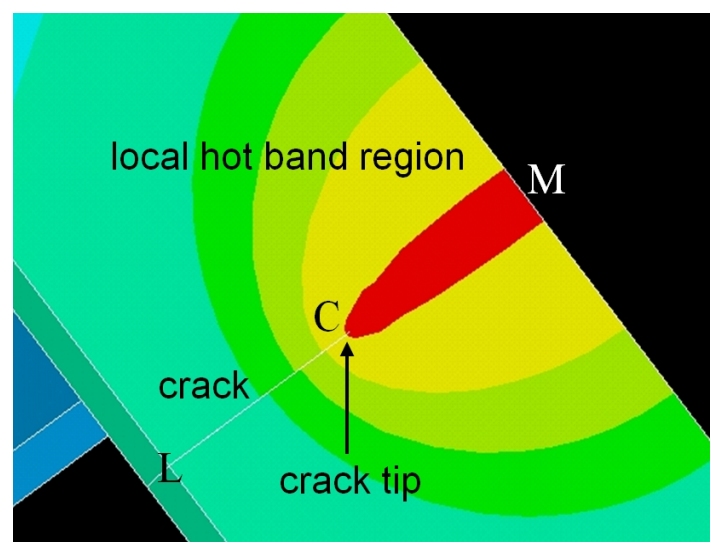

Figure 7: Hot band region in front to crack tip.

chanical contact condition along the crack surfaces.

\section{Results and Discussions}

\subsection{Hot Region near Crack Tip In this research, the} geometric parameters are as follows:

(1) $l=3.8 \mathrm{~mm}, h=2.42 \mathrm{~mm}$

(2) $R_{1}=R_{2}=0.5 \mathrm{~mm}, R_{3}=0.3 \mathrm{~mm}$

(3) $e=0.1 \mathrm{~mm}, w=1 \mathrm{~mm}$

(4) $a=0.5 \mathrm{~mm}, b=1 \mathrm{~mm}$

In addition, the heat transfer parameters for the heat transfer are as follows:

(1) $T_{0}=T_{\infty}=26{ }^{\circ} \mathrm{C}$

(2) Convection coefficient: $h_{c}=21 \mathrm{~W} / \mathrm{m}^{2}-K$

As a result, Fig. 6 Fig. 9 show the temperature field and electric current density under $I_{0}=20 \mathrm{~A}$ at $0.25 \mathrm{~s}$. It can be seen that the Joule heating effect causes a high temperature area (local hot band region) in front to the crack tip. Also, there is a local concentration of the electric current density near the crack tip. Similar to the elastic stress field, the electric current density also has the $r^{-1 / 2}$ singularity at the crack tip [2].

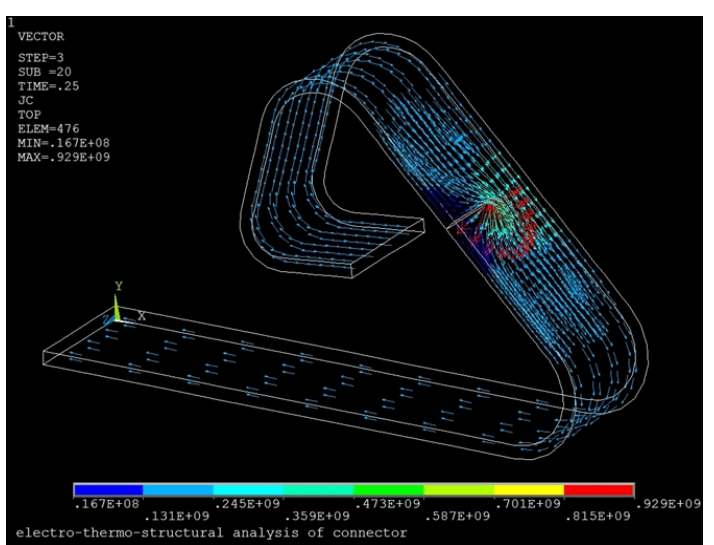

Figure 8: Electric current density field (units: $A / \mathrm{m}^{2}$ ).

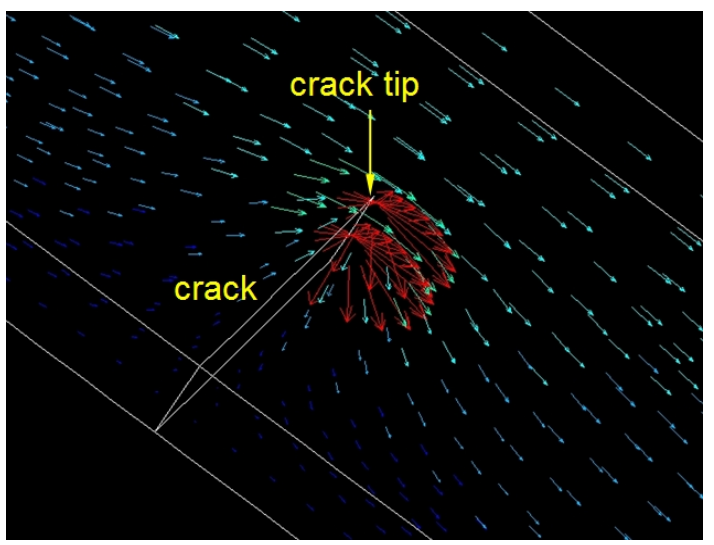

Figure 9: Electric concentration around crack tip.

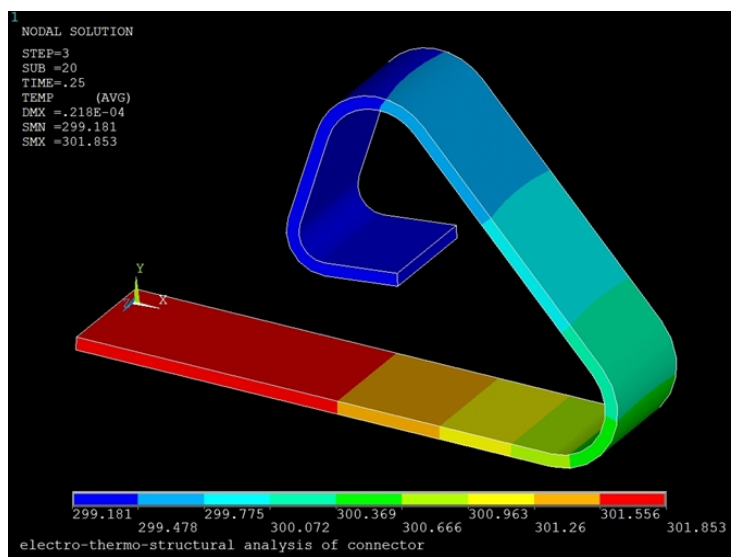

Figure 10: Temperature distribution without crack (units: ${ }^{\circ} \mathrm{C}$ ).

3.2 Effect of Crack Figure 10 shows the temperature distribution without the crack. There is no local hot region in this case. However, in Fig. 7, it proves that the local hot region can be used to determine the crack tip location. This concept can be applied to the crack/defect detection by the use of the thermal camera and image.

3.3 Effect of Electric Operating Time In this section, the condition $I_{0}=20 \mathrm{~A}$ is used. Referring to Fig. 7, the temperature distribution along the path LCM is discussed. For the discussion, the temperature ratio $T / T_{L}$ is defined, 


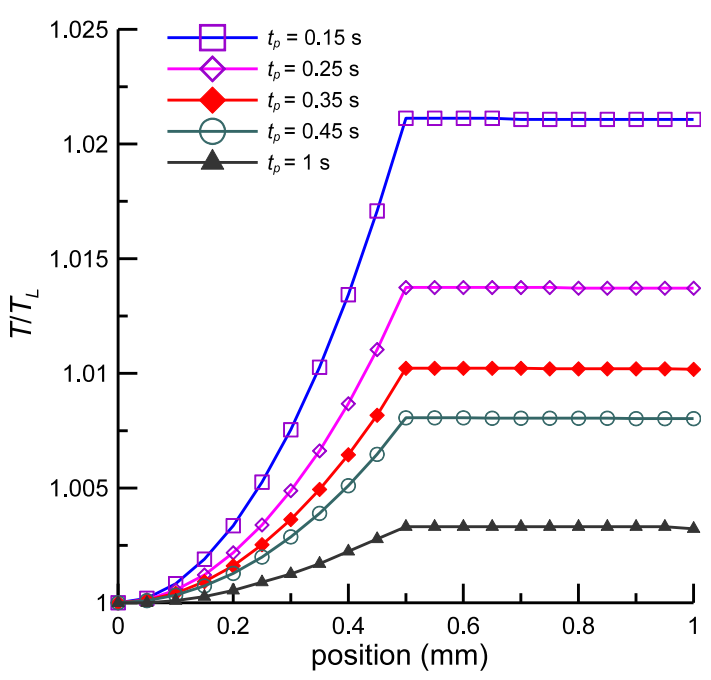

Figure 11: Distribution of $T / T_{L}$ under different $t_{p}$.

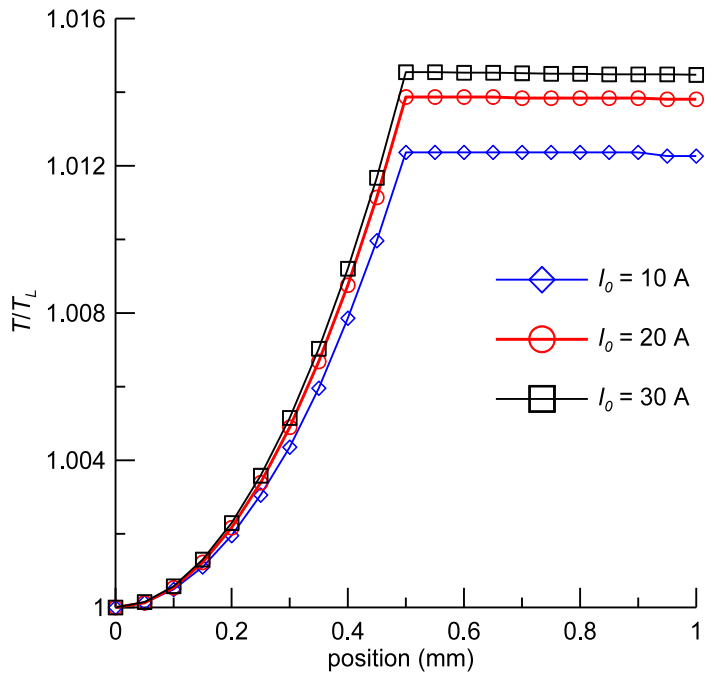

Figure 12: Distribution of $T / T_{L}$ under different $I_{0}$.

where $T_{L}$ is the edge temperature at the point $L$. The larger value of $T / T_{L}$ at the crack tip implies the larger temperature difference between the crack tip and environment. Above situation is better for the crack detection.

In Fig. 11, it shows the distribution of $T / T_{L}$ along the path LCM under different operating times $\left(t_{p}\right)$. The values of $T / T_{L}$ almost keep constant along the position $0.5 \sim 1 \mathrm{~mm}$, i.e. the path $\mathrm{CM}$. As a result, the crack tip location (point $C)$ can be determined by the Fig. 11 . When the electric operating time $t_{p}$ is shorter, $T / T_{L}$ at the crack tip is larger. The shorter electric operating time is better for the crack detection.

3.4 Effect of Electric Current In this section, the condition $t_{p}=0.25 \mathrm{~s}$ is used. In Fig. 12, it shows $T / T_{L}$ distribution under different $I_{0}$. The curves are similar to Fig. 11. When the electric current $I_{0}$ is larger, $T / T_{L}$ at the crack tip is larger. Larger electric current is better for the crack detection.

3.5 Optimal Condition for Crack Detection According to previous sections, two better loading conditions

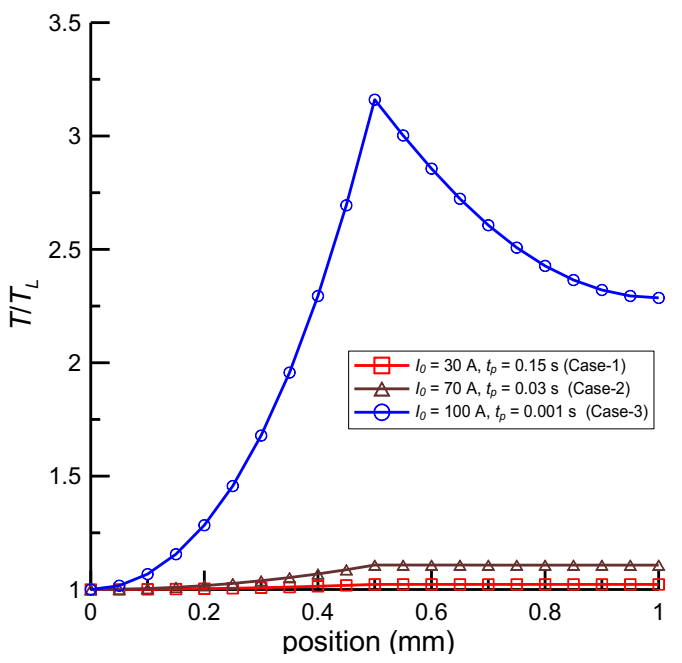

Figure 13: Distribution of $T / T_{L}$ of three cases.

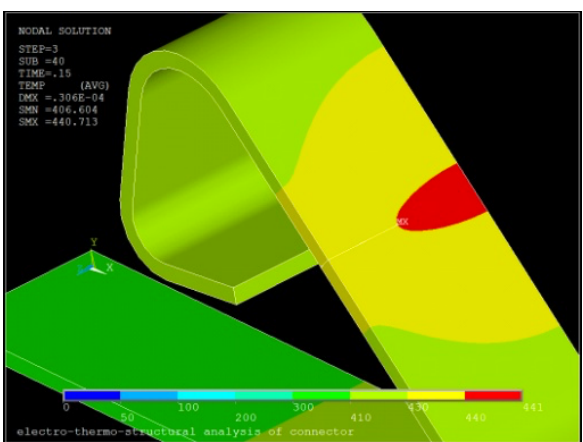

(a) Case-1

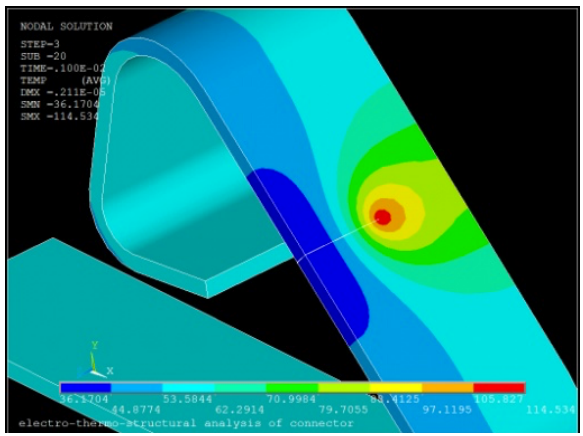

(b) Case-3

Figure 14: Temperature contours.

for the crack detection are found as follows: (a) shorter electric operating time, (b) larger electric current. Based on above conclusions, the following three cases are adopted for the further analysis:

(1) Case-1: $I_{0}=30 \mathrm{~A}, t_{p}=0.15 \mathrm{~s}$

(2) Case-2: $I_{0}=70 \mathrm{~A}, t_{p}=0.03 \mathrm{~s}$

(3) Case-3: $I_{0}=100 \mathrm{~A}, t_{p}=0.001 \mathrm{~s}$

In Fig. 13, it shows the distribution of $T / T_{L}$ of three cases. Obviously, a peak value of $T / T_{L}$ occurs at the crack tip in Case-3. Also, this case has larger values of $T / T_{L}$ than 
other two cases. It implies that the loading condition of Case-3 is optimal case in this analysis.

From the temperature contours in Fig. 14, it shows the advantage of the Case- 3 condition. In Case- 3 , a local hot spot occurs at the crack tip and it can be used to determine the crack tip location precisely. However, in Case-1, a hot band region in front to crack tip is observed. For the crack detection, the band region is not as good as the hot spot.

\section{Conclusion}

The crack detection technique with the Joule heating effect is numerically investigated in this paper. For the accurate crack detection, two better electric loading conditions are found as follows: (a) shorter electric operating time $\left(t_{p}\right)$, (b) larger electric current $\left(I_{0}\right)$. The optimal condition, $I_{0}$ $=100 \mathrm{~A}$ and $t_{p}=0.001 \mathrm{~s}$, is found for this crack detection research.

\section{Acknowledgment}

The author is grateful to Ming Chi University of Technology (MCUT) for the financial support under contract numbers VL000-1100-108 and VL009-1100-110. Also, the author would like to thank the Center for Reliability Engineering in MCUT for additional financial support.

\section{References}

[1] V. Z. Parton and B. A. Kudryavtsev, "Electro-magneto- elasticity", Gordon and Breach, New York, 1988.

[2] G. X. Cai and F. G. Yuan, "Electric current-induced stresses at the crack tip in conductors", International Journal of Fracture, Vol.96, pp.279-301, 1999. DOI: 10.1023/A:1018670829822

[3] D. Hasanyan, L. Librescu, Z. Qin and R. D. Young, "Thermoelastic cracked plates carrying nonstationary electrical current”, Journal of Thermal Stresses, Vol.28, pp.729, 2005.

[4] T. J. C. Liu, "Crack detection/arrest with Joule heating", Encyclopedia of Thermal Stresses, Hetnarski, R.B. (Ed.), Springer Science + Business Media Dordrecht, Dordrecht, 2013. DOI: 10.1007/978-94-007-2739-7_303

[5] T. J. C. Liu, "Joule heating behaviors around through crack emanating from circular hole under electric load", Engineering Fracture Mechanics, Vol.123, pp.2-20, 2014. DOI: 10.1016/j.engfracmech.2014.01.016

[6] Online Materials Information Resource - MatWeb, http: //matweb.com/, access date: 2021.04.22

[7] "ANSYS 14.0 Mechanical APDL theory reference", SAS IP, Inc./ANSYS, Inc., USA, 2011.

[8] X. Sun and P. Dong "Analysis of aluminum resistance spot welding processes using coupled finite element procedures", Welding Journal, Vol.79, pp.s215, 2000. DOI: 10.1034/j.1600-0692.2000.d01-21.x

[9] C. L. Tsai, W. L. Dai, D. W. Dickinson and J. C. Papritan, "Analysis and development of a real-time control methodology in resistance spot welding", Welding Journal, Vol.70, pp.s339, 1991.

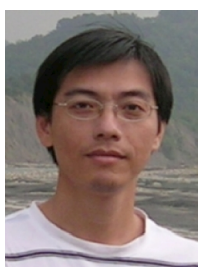

Thomas Jin-Chee Liu (Member) is an Associate Professor in Department of Mechanical Engineering, Ming Chi University of Technology. He received the Ph.D. degree in Mechanical Engineering from National Cheng Kung University in 2005. His research interests are fracture mechanics, solid mechanics, finite element analyses, and computer-aided engineering (CAE). 